\title{
CORRECTION
}

\section{Correction: Molecular dynamics simulation study of distribution and dynamics of aqueous solutions of uranyl ions: the effect of varying temperature and concentration}

Cite this: Phys. Chem. Chem. Phys., 2016, 18, 1344

\author{
Manish Chopra ${ }^{a}$ and Niharendu Choudhury ${ }^{\star b}$
}

DOI: $10.1039 / c 5 c p 90224 j$

Correction for 'Molecular dynamics simulation study of distribution and dynamics of aqueous solutions of uranyl ions: the effect of varying temperature and concentration' by Manish Chopra et al., Phys.

www.rsc.org/pccp

Chem. Chem. Phys., 2015, 17, 27840-27850

The authors would like to correct the units provided for the diffusion coefficients in Tables 2, 3, 4 and 5 of the published article which should read as $10^{-5} \mathrm{~cm}^{2} \mathrm{~s}^{-1}$, as seen in the amended Tables below.

Table 2 Diffusion coefficient values of overall water molecules at different temperatures for U1, U5 and U10 systems

Diffusion coefficients, $D_{\mathrm{PBC}}\left(10^{-5} \mathrm{~cm}^{2} \mathrm{~s}^{-1}\right)$ of overall water molecules

\begin{tabular}{llllll}
\hline & $240 \mathrm{~K}$ & $270 \mathrm{~K}$ & $298 \mathrm{~K}$ & $330 \mathrm{~K}$ & $360 \mathrm{~K}$ \\
\hline U1 & 1.82 & 3.43 & 5.28 & 7.51 & 9.60 \\
U5 & 1.59 & 2.98 & 4.50 & 8.42 & 4.88
\end{tabular}

Table 3 Diffusion coefficient values of water molecules within first coordination shell of uranyl ions at different temperatures for U1, U5 and U10 systems

Diffusion coefficients, $D_{\mathrm{PBC}}\left(10^{-5} \mathrm{~cm}^{2} \mathrm{~s}^{-1}\right)$ of coordination shell water molecules

\begin{tabular}{llllll}
\hline & $240 \mathrm{~K}$ & $270 \mathrm{~K}$ & $298 \mathrm{~K}$ & $330 \mathrm{~K}$ & $360 \mathrm{~K}$ \\
\hline U1 & 0.56 & 0.93 & 1.86 & 2.19 & 2.94 \\
U5 & 0.54 & 0.86 & 1.35 & 2.09 & 2.44 \\
U10 & 0.43 & 0.75 & 1.20 & 1.61 & 1.94
\end{tabular}

Table 4 Diffusion coefficient values for uranium atom of uranyl ions at different temperatures for U1, U5 and U10 systems

Diffusion coefficients, $D_{\mathrm{PBC}}\left(10^{-5} \mathrm{~cm}^{2} \mathrm{~s}^{-1}\right)$ for uranium atom of uranyl ion

\begin{tabular}{lllllr}
\hline & $240 \mathrm{~K}$ & $270 \mathrm{~K}$ & $298 \mathrm{~K}$ & $330 \mathrm{~K}$ & $360 \mathrm{~K}$ \\
\hline U1 & 0.46 & 0.76 & 1.37 & 1.89 & 2.43 \\
U5 & 0.36 & 0.70 & 1.09 & 1.63 & 2.12 \\
U10 & 0.29 & 0.55 & 0.87 & 1.19 & 1.46
\end{tabular}

\footnotetext{
${ }^{a}$ Radiation Safety Systems Division, Bhabha Atomic Research Centre, Mumbai - 400 085, India

${ }^{b}$ Theoretical Chemistry Section, Bhabha Atomic Research Centre, Mumbai - 400 085, India. E-mail: nihcho@barc.gov.in; Fax: +91 22-2551 5151; Tel: +91 22-2559 5089
} 
Table 5

Diffusion coefficient values of water molecules and uranyl ions corrected for system size at different temperatures for U1, U5 and U10 systems

\begin{tabular}{|c|c|c|c|c|c|}
\hline & $240 \mathrm{~K}$ & $270 \mathrm{~K}$ & $298 \mathrm{~K}$ & $330 \mathrm{~K}$ & $360 \mathrm{~K}$ \\
\hline \multicolumn{6}{|c|}{ Corrected diffusion coefficients, $D_{\mathrm{o}}\left(10^{-5} \mathrm{~cm}^{2} \mathrm{~s}^{-1}\right)$ of overall water molecules } \\
\hline U1 & 2.25 & 4.01 & 6.07 & 8.59 & 10.98 \\
\hline U5 & 2.02 & 3.56 & 5.29 & 7.50 & 9.39 \\
\hline U10 & 1.79 & 3.03 & 4.37 & 5.96 & 7.65 \\
\hline \multicolumn{6}{|c|}{ Corrected diffusion coefficients, $D_{\mathrm{o}}\left(10^{-5} \mathrm{~cm}^{2} \mathrm{~s}^{-1}\right)$ of coordination shell water molecules } \\
\hline U1 & 0.99 & 1.51 & 2.65 & 3.27 & 4.32 \\
\hline U5 & 0.97 & 1.44 & 2.26 & 3.17 & 3.82 \\
\hline U10 & 0.86 & 1.33 & 1.99 & 2.69 & 3.32 \\
\hline \multicolumn{6}{|c|}{ Corrected diffusion coefficients, $D_{\mathrm{o}}\left(10^{-5} \mathrm{~cm}^{2} \mathrm{~s}^{-1}\right)$ of uranium atom of uranyl ions } \\
\hline U1 & 0.89 & 1.34 & 2.16 & 2.97 & 3.81 \\
\hline U5 & 0.79 & 1.28 & 1.88 & 2.71 & 3.50 \\
\hline U10 & 0.72 & 1.13 & 1.66 & 2.27 & 2.84 \\
\hline
\end{tabular}

In addition, on page 27848,4 th line above Table 5 , the value $2.3 \times 10^{-9} \mathrm{~cm}^{2} \mathrm{~s}^{-1}$ should actually be $2.3 \times 10^{-5} \mathrm{~cm}^{2} \mathrm{~s}^{-1}$. The Royal Society of Chemistry apologises for these errors and any consequent inconvenience to authors and readers. 